\title{
Edge enhancement of IBP reconstruction by using sharp infinite symmetrical exponential filter
}

\author{
A.R.A Nazren ${ }^{1}$, Ngadiran. $\mathbf{R}^{2}$, S.N. Yaakob ${ }^{3}$ \\ ${ }^{1}$ Embedded Computing Research Cluster, University Malaysia Perlis (UniMAP), Malaysia \\ ${ }^{2,3}$ School of Computer and Communication Engineering of University Malaysia Perlis (UniMAP), Malaysia
}

\begin{tabular}{l}
\hline Article Info \\
\hline Article history: \\
Received Sep 27, 2018 \\
Revised Nov 28, 2018 \\
Accepted Dec 2, 2018 \\
\hline
\end{tabular}

\section{Keywords:}

Iterative back projection ISEF

Ringing artefacts Edge enhancement

Super resolution

\begin{abstract}
This study contributes to provide an enhancement technique for improving the Iterative Back Projection (IBP) Super Resolution technique by using the Sharp Infinite Symmetrical Filter (SISEF). Theoretically, the IBP technique operates as minimizer the error reconstruction iteratively until it refined the High Resolution (HR) image. However, due to iterative manner and lack of edge guidance during the back projection operation, this technique has suffered from produced the ringing artefact on the HR image appearances. Additionally, the IBP reconstruction also demands for large number iteration for accomplishing the prediction HR image. This problem arose when the IBP estimator tended to oscillate at the same solution frequently. In order to overcome these constraints, the SISEF is deployed as regulator to improve the IBP estimator with provides an accurate edge information for enhancing the edge image and reduce the ringing artefacts. Fortunately, highly precision of edge information provided by SISEF capable to reduce amount of estimation process repetition.
\end{abstract}

Copyright (C) 2019 Institute of Advanced Engineering and Science. All rights reserved.

\section{Corresponding Author:}

Amir Nazren Abdul Rahim,

School of Computer and Communication Engineering,

Universiti Malaysia Perlis (UniMAP),

Pauh Putra Campus, 02600 Arau Perlis, Malaysia.

Email: amirnazren@unimap.edu.my

\section{INTRODUCTION TO SUPER RESOLUTION ENHANCEMENT}

The area of this study is getting a lot of interests from researcher since the conventional Super Resolution (SR) technique suffers from imperfection in the High Resolution (HR) image estimation. In Chiang et al [1] works, an improvement of IBP is produced in the resampling algorithm by integrating the image warping algorithm inside the IBP technique. Moreover, they proposed the implementation of coupling the degradation model of imaging system into integrating resample to produce better estimation. In Cohen et al [2] work, an improvement is suggested through applying Polyphase filter to replace the geometric transformation inside the Iterative Back Projection (IBP) and improved the image resolution. Furthermore, the edge enhancement technique proposed by Hyunwoo et al [3] using an Anisotropic Diffusion to suppress the noise and highlighted the edges of image.

On top of that, the image enhancement is done in Yan et al [4] works. The combination of PapoulisGerchberg extrapolation inside the IBP reconstruction is proposed to enhance the spiral Computed Tomography (CT) slice image. Besides that, Qin suggested the efficient improvement in the IBP algorithm through improvement of the first initial guess which is estimated by wavelet locally adaptive algorithm compared to normal interpolation method [5]. Next Liang et al [6] also gave improvement in the edge detection during conducting the initial interpolated image via apply Non-Local IBP fast algorithm. In the Bengtsson et al [7] proposed L*a*b domain for improving the Human Visual System in the HR image. This technique is capable to improve the output of HR image by preserving the edge information. 
In order to recover the HR image, Bareja et al [8] proposed a combination of IBP algorithm with Canny Edge Detector and error difference image to recover loss of frequency information. The improvement is continued in Nayak et al [9], by proposing a hybrid technique between IBP technique and Cuckoo optimizer to enhance the initial guess and HR output image. In Pejman et al work [10], the improvement in the IBP algorithm is proposed through applying the interpolation of LR images before registered inside the IBP algorithm. Another work in Cheref et al [11] introduced edge enhancement by using the Anisotropic Diffusion technique to preserve any possible edge image. In Mei et al [12] works, implementation of adaptive Perona Malik as edge preservation is done in SR reconstruction.

The enhancement works in the IBP is continued with reducing the ringing artefacts by applying a variant and local variance variant based on adaptive analysis [13]. Moreover, the quality of visual output reconstruction image is improved through applying the sparse linear regression and IBP. Additionally, modification in Gaussian high pass filter is done to refine the initial guess [14]. Furthermore, the accuracy of reconstruction can be increased when the residual error is enhanced by implementing the Anisotropic Diffusion with the help of IBP estimator to produce fine HR image with edge preservation technique [15]. In Makwana et al [16] proposed a combination of IBP algorithm with Canny edge detector and Gabor filter for improving the error minimization during back projection operation. Besides that, Nayak et al [17] provided the edge image improvement in the IBP technique by using the P-Spline and MuCSO-QPSO algorithm. The P-Spline provides sharp edge information and MuCSO-QPSO optimized the estimation method.

The Iterative Back Projection technique has ability to minimize the reconstruction error efficiently in iterative manner. This error reconstruction is back projecting to HR grid to correcting the next estimation image. Although this technique improves the image intensity with removing the blur effect. However, the iterative routine tends to produce the ringing artefacts along the edges because of back projecting is without any edge guidance. In the present work, this study proposes the edge enhancement technique inside the Iterative Back Projection technique. This edge enhancement technique is combined between the IBP reconstruction and Sharp Infinite Symmetrical Exponential Filter (SISEF). The SISEF function as sharp edges detection and then preserves the possible edges detected by re-highlights the edges inside the estimation process. Coincide, the information supplies works as edge guidance during the back projection to avoid across edge projection thus the ringing effects can be removed. This sharp edge detected capable to enhance the reconstruction HR estimation with reduce the error reconstruction and preserving the lost frequency information with robust toward ringing effects and rapid estimation process.

\section{RESEARCH METHOD}

\subsection{Iterative Back Projection}

The main idea of the Iterative Back Projection (IBP) technique is to reconstruct the High Resolution (HR) image from observing the multiple degraded input images. The estimation done by projecting back the difference between simulated Low Resolution (LR) images and the observed LR images [18]. The estimation keeps continued until the HR image generated which has same LR images with observed LR images after passing the same blurring and down sampling process. Basically the IBP method can be divided into two phases. In the first phase, it generates the simulated image and then projected back the error from the difference between simulated and observed LR images to reconstruct the HR estimation [19]. The construction of simulated the LR images $Y_{k}$ can be formulated in 1 .

$$
Y_{k}=D_{k} B_{k} W_{k} f
$$

This simulated LR image $Y_{k}$ has number $k^{\text {th }}$ of image and the pixel intensity of LR images derived from the HR image $f$ after get influenced by the degradation function. The degradation function is a combination of blur kernel function $B_{k}$ and translation wrapping function $W_{k}$ influenced before getting the down sampling by the decimation operator $D$.

In the reconstruction of IBP process, this technique starts with computing the initial guess $f^{(0)} \mathrm{HR}$ image via applies the average method. Then, the imaging process determines the set of simulated LR image $Y_{k}{ }^{(0)}$. However, the initial guessing process may provide many solutions than one accurate solution. The selection of accurate the initial guess method could driving the algorithm to determine an unique, faster and smooth solution. A good selection of an initial guess method was suggested by Irani is the average method of the attributes of information from registered LR images observed [18] such in the 2.

$$
f^{(0)}=\frac{1}{N} \sum_{k=1}^{N} W_{k}^{-1}\left(Y_{k}\right)
$$

\footnotetext{
Edge enhancement of IBP reconstruction by using sharp infinite symmetrical exponential... (A.R.A Nazren)
} 
If the initial guess HR image $f^{(0)}$ is matching enough with the reference image, then the simulated images $Y_{k}^{0}=D_{k} B_{k} W_{k} f^{(0)}$ should be identical with the observed images $Y_{k}$ and no more correction is required. If the difference images arise between $Y_{k}$ and $Y_{k}{ }^{0}$, then the improvement the initial guess by back projecting the error with combining the current information in the HR grid. This step is yielding an improvement to the next HR image $f^{(1)}$ such expressed in the 3.

$$
f^{t+1}=f^{t}+\frac{1}{N} \sum_{k=1}^{N} Q_{k}^{T}\left[Y_{k}-Q_{k} f^{t}\right]
$$

Where

$$
Q_{k}=D_{k} B_{k} W_{k}
$$

This procedure keeps repeating in iterative manner until it meets the local minimum error. The error function can be described as in 5 .

$$
e^{t}=\sqrt{\frac{1}{N} \sum_{k=1}^{N}\left\|Y_{k}-Y_{k}^{t}\right\|_{2}^{2}}
$$

\subsection{The Infinite Symmetrical Exponential Filter (ISEF)}

The Infinite Symmetrical Exponential Filter or Shen Castan Edge Detector is an optimal smoothing filter with a very simple recursive algorithm. The core concept of this filter is implementing the differentiation of optimal exponential. It gives a good impact during edge detection when it has less responds to noise and give precision in edge localization. Generally, the problem facing of Laplacian Gaussian filter during the edge detection which it has contradiction between the noise insensibility and the precision localization. Thus to overcome this problem, the ISEF proposed the linear filter based on one step model and multi edge detection.

This optimal smoothing filter is a symmetric exponential in infinite large window size and very simple recursive algorithm. The difference between input and output of this recursive filter has the capability to detect the edges with less sensitivity to noise and high precision in localization. The ISEF filter has proposed two methods for edge detection either can be used the maxima of the gradient (GEF) or the zero crossing of second directional Derivative along the Gradient (SDEF). The ISEF filter produced the GEF and SDEF from the first and second derivatives of the operator for symmetric exponential filters respectively. Initially, the normalized symmetric exponential filter in one dimensional (1D) is written in 6.

$$
f_{L}(x)=C\left(f_{1}(x)+f_{2}(x)-a_{0} \delta(x)\right)
$$

Where

$$
C=\frac{1}{\left(2-a_{0}\right)}
$$

The exponential is a symmetrical function which is can be divided into the right side function $f_{1}(x)$ and the left side function $f_{2}(x)$. Thus the second derivative of exponential function for the SDEF along the $\mathrm{x}$ axis can describe in 8 .

$$
I_{x x}(x, y)=\frac{\partial^{2}}{\partial x^{2}}(I(x, y) * f(x, y))
$$

Where in the discrete form of SDEF is written in 9.

$$
I_{x x}(x, y)=I(x, y) * f_{1}(y) * f_{2}(y) *\left(f_{1}(y)+(y)\right)-2\left(I(x, y) * f_{1}(y) * f_{2}(y)\right)
$$

The equation above also can be used to calculate the gradient along the $\mathrm{y}$ axis direction. For instance, this equation scheme can be illustrated in the process flow Figure 1.

\subsection{Previous Works in Edge Enhancement Technique}

The edge enhancement technique provides additional correction information during the reconstruction computation based on the guided edge information detected. The information of edge 
detected, then is used to strengthen back the lost edge information. Additionally, this process provides an edge guided to the IBP technique during the back projecting to HR grid size to avoid a ringing effect disturbance. Furthermore, the efficiency of reconstruction process is influenced by the precision the edge detection provided. This high precision of localization and high rate detection are important features to increase the rate of edge detection and at the same time, it helped to increase the rate of correction process. This study meets two good technique have implemented with IBP in edge enhancement technique such Anisotropic Diffusion and ISEF filter.

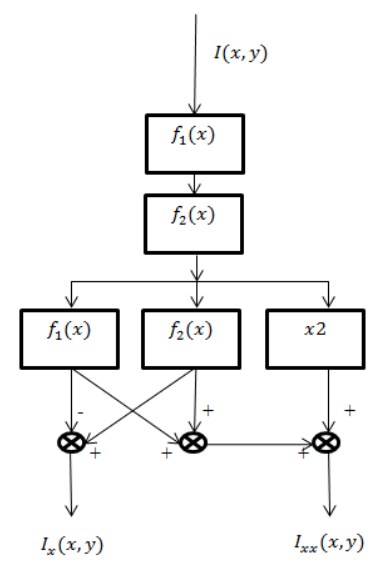

Figure 1. ISEF process flow

\subsection{Propose IBP technique with Sharp ISEF regulator}

This study has concerned on the edge detection is provided by the ISEF method. This Shen-Castan method has some advantages such as yields edge detail information with less responds to noise and high precision edge localization. This study also interests to use edge detection through zero crossing provided by the Second Directional Derivative Fillter (SDEF). This filter is based on exponential function which gives better performance than the Gaussian based filter. Hence, this technique could provide much better edge preserving [20]. The high accuracy in SDEF detection supplies much possible additional information especially about high frequency information. This precision on information helps the estimator to make predictions and preserves the weak and loss of edges more efficient.

As aforementioned earlier, the IBP method performed well in minimizing the residual error during the HR estimation. The estimation is done by back projecting the difference of observed image and simulated image to the HR correction process. This iterative process keeps on repeating until no more error cost function arises without any edge guidance. Consequently, estimated the HR by the IBP algorithm is not able to fully recover the lost edge information. Thus, the ISEF method is the best solution to improve the deblurring process and sharpen the edge details. This ISEF implementation can be illustrated as in the Figure 2.

According to the proposed algorithm in Figure 3, the initial guess gets the first enhancement from the implemented ISEF. This process is important since the observed LR will be magnified to the HR grid, thus some information will be lost and blurry image is generated. Also, this stage gives improvement in the quality of initial guess image to get close to the possible to optimum estimation. As the HR initial guess has good quality, the IBP reconstruction only requires few efforts for enhancing process. Besides increasing the quality of HR estimation, this stage also contributes to save a lot if resources in a faster way. The first refine process in the initial guess can be expressed in 10.

$$
f^{0}=f^{0}+\lambda\left(f^{0} * H_{I S E F}\right)
$$

Where $\lambda$ is constant for the stability equation. $f^{0}$ is the first initial guess projected from the observed LR images by using the average method. In order to enhance the $f^{0}$, the SDEF method in the ISEF observes the priori information initial guess to produce the gradient information. This gradient contains an edge information which is then fused inside the current estimation image. This additional edge information provides recovering of the weaker frequency information and strengthens back the structure image.

Edge enhancement of IBP reconstruction by using sharp infinite symmetrical exponential... (A.R.A Nazren) 
Basically, the residual information is generated from the difference of simulated and observed LR images which are used to correct the current HR estimation. Meanwhile, the correction process can be optimized when adding the extra edge information. In Patel [21] work, the additional gradient information is directly fused inside the residual information. However, this approach has some differences since additional information needs a step size or weighting factor to ensure this combination converges to the optimal local minima faster. The suitable value of step size is crucial criteria to ensure the estimation achieves optimum HR output. Besides that, it also gives improvement in the number of repetitions on of the system. This calculation for step size can be referred in 11 .

$$
0<\tau<\frac{2}{\sup _{x}\|H f(x)\|}
$$

Where $H f(x)$ is the Hessian function of matrix of $f$ at $x$ and $\|\cdot\|$ is the spectral operator norm which used to find the largest eigenvalue of function $f$. The parameter $\tau$ is known as the step size for gradient of image edges computed.

In order to determine the optimal result, this optimal residual information is then projected back to HR grid. This scenario can be described in mathematical as such 12 .

$$
f_{t+1}=f_{t}+\frac{1}{N} r^{t}+\tau\left[\left(f-f_{t}\right) * I S E F-\left(f_{t-1}\right) * I S E F\right]
$$

In Patel works, the enhancement of the edges is taken from the difference of computed initial guess and current HR. This difference provides a guidance to the edge intensity and the precision in the edge localization. However, the precision edge localization can be increased by shrinking the width edges to become sharper. Thus, the accuracy in edge localization contributes to more accurate estimation, as well as an increment in the quality of output and decrease the number of iterations for estimation process. The sharper edge process can be done by subtracting the difference edge $\left(f-f_{t}\right)$ with edge detected from the previous state HR image $\left(f_{t-1}\right)$. Both of detected edges are determined by the SDEF method just before the subtraction operation. This situation can be illustrated such in Figure 2.

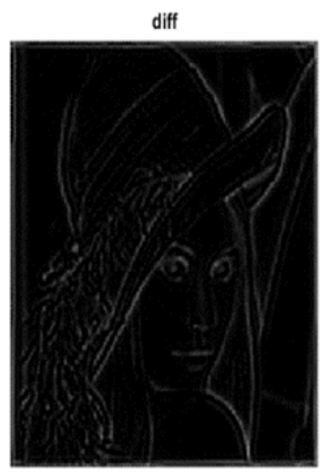

(a)

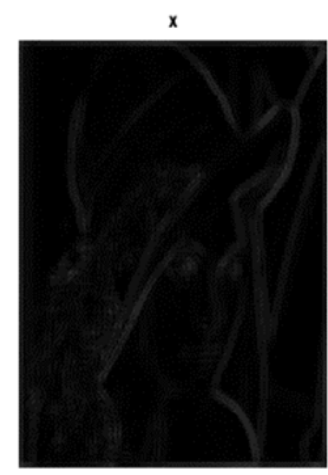

(b)

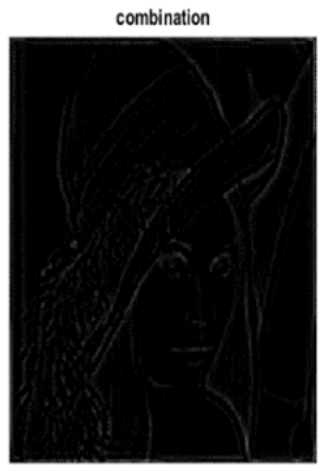

(c)

Figure 2. Sharper gradient image (a) difference edge of HR (b) edge of priori HR (c) proposed sharp edge

\section{RESULTS AND ANALYSIS}

This section presents the experiments and results obtained by the proposed combination between IBP with SISEF edge enhancement technique. These experiments use the MATLAB platform as experiment simulator. These experiments also use the standard image form ISI and DESCAI database with the standard image size is $256 \times 256$ resolution. Firstly, this experiment creates a sequence four LR images. Each of LR images has shifted from original image by a pixel in the vertical direction. Then, these images applied effect of camera PSF. The effects involved in this process contain of blurry and decimation operation. Then this shifted LR image convoluted with the Gaussian low pass filter of size $3 \times 3$ with a standard deviation of 3 . Then resulting image was down sampling by factor 2 in each direction. Therefore, the same operation with the different motion vectors in vertical and horizontal directions is used to produce four LR images. The proposed technique measurement based on the highest PSNR recorded. Besides that, the cost 
computation complexity measurement is used based on number of iterations consuming for finishing the HR estimation. The results and analysis are equipped the comparison with the Cheref work [11] and Patel work [21] along the work.

\subsection{Situation: Blurry Effect Information Perfectly Known}

The result in Table 1 shows that the combination IBP with proposed technique Sharp ISEF regulator (SISEF) is leading for most of PSNR results. By looking at the average result, this technique gives an improvement about $1.75 \mathrm{~dB}$ compares than ordinary IBP technique and this result is a higher quality improvement is provided compare to other techniques. This means, the SISEF proposed technique has the capability to provide a better edge enhancement. This edge detector equipped with higher localization precision and less error detection, therefore in most cases of image tested this proposed ISEF has produced a better performance.

Besides that, by comparing with the Patel technique. This proposed technique successfully to increase the PSNR up to $0.758 \mathrm{~dB}$ than Patel technique. Furthermore, by referring in Figure 3, this SISEF has successfully increased the precision and accuracy of edge priori information from the conventional ISEF method in Patel work. Thus, this contribution helps the IBP estimator predicts the HR construction more precisely and accurately. Additionally, Figure 4 has shown the SISEF has successfully reduced the ringing artefacts around the edges of the HR image compared with the ordinary IBP technique. Perhaps, this situation helps to increase the appearance of HR image.

Despite of the good performance, this technique has difficulty during handling the critical image such the image contains a lot of high frequency information. For example, the Mandrill image contains a lot of high frequency information such hair around the mandrill face. This disadvantage rose when the SISEF detector only traces the edges in horizontal and vertical direction only. Hence, in some direction this detector failed to provide details high frequency information.

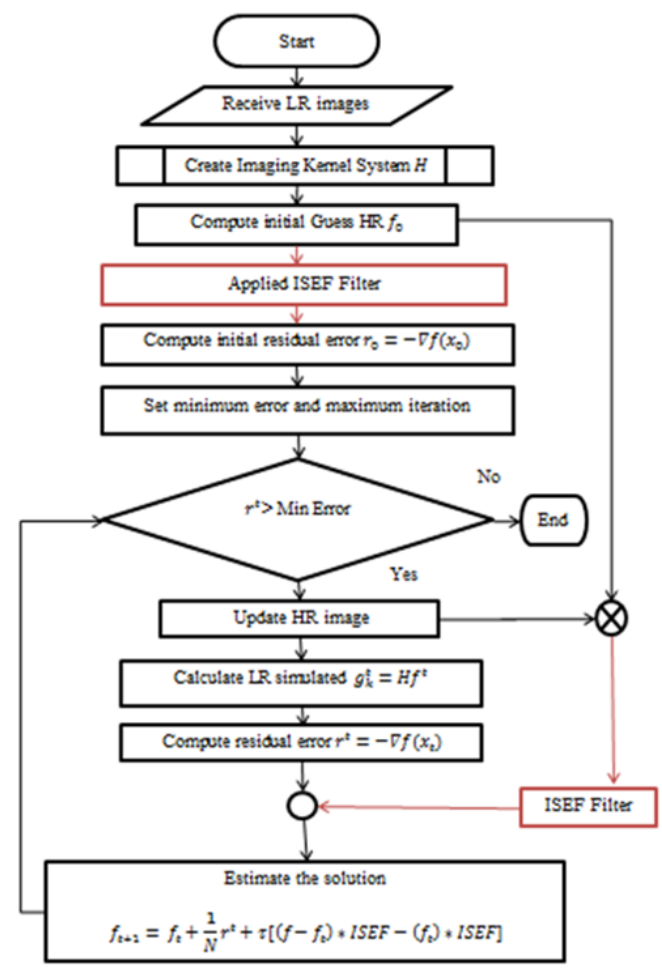

Figure 3. The IBP with lorentzian ISEF regulator process flow 


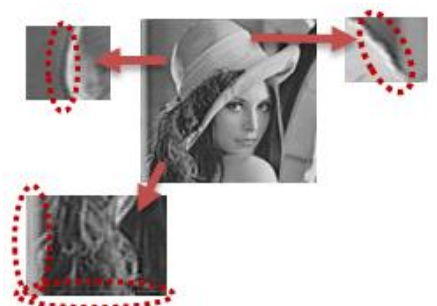

(a)

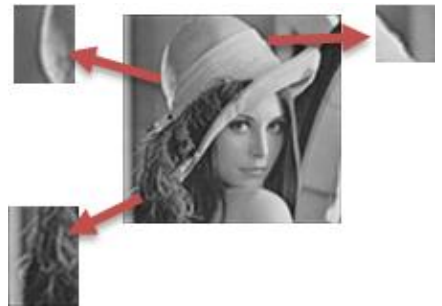

(b)

Figure 4. Ringing artefacts (a) IBP output contaminated with the ringing artefacts (b) proposed technique has reduced the ringing artefacts

Table 1. PSNR for Multi Kinds of Input Image

\begin{tabular}{llllll}
\hline & \multicolumn{4}{c}{ The IBP technique with } & \\
& IBP & Patel & Cheref & CANNY & SISEF \\
\hline LENA & 27.730 & 28.651 & 28.034 & 28.018 & 28.952 \\
CAMERAMAN & 26.477 & 27.220 & 27.319 & 26.758 & 27.224 \\
MANDRIL & 23.284 & 23.464 & 23.486 & 23.336 & 23.273 \\
PEPPER & 25.941 & 27.956 & 29.023 & 26.391 & 30.253 \\
CLOWN & 24.560 & 26.673 & 27.932 & 25.067 & 28.784 \\
JETPLANE & 26.020 & 27.177 & 27.571 & 26.353 & 27.648 \\
BRAIN & 28.041 & 30.131 & 31.319 & 28.699 & 32.533 \\
SHIP & 28.923 & 29.494 & 29.714 & 29.188 & 29.435 \\
USAF & 22.670 & 22.183 & 22.624 & 22.656 & 22.183 \\
BRIDGE & 23.982 & 24.648 & 24.824 & 24.178 & 24.890 \\
\hline
\end{tabular}

On the other hand, in some cases the technique proposed by Cheref also provides the highest quality. This technique performed well during handling difficult image such Mandrill and USAF image. The Anisotropic Diffusion in Cheref works has the capability to seek the edge in many directions (north, south, west, and east). Thus, this method has the capability to provide the detail edge information and give a promising result in the difficult image. Moreover, this edge detector has good rate detection and minimum responds toward reduction edges. Thus, this technique is among the best edge enhancement technique.

The Gaussian based filter design such as Canny techniques only provides a little improvement. This condition happens when the Gaussian based filter has a contradiction function between providing smooth function and precise localization. Furthermore, the Canny edge detector can provide a very sharp edge image, but the intensity edge detected is small. Normally, this type of filter has less precise localization than ISEF filter.

\subsection{Number of Iterations}

In terms of the computational complexity, the IBP with edge enhancement technique measurement is based on the number of iteration algorithm for accomplishing the estimation. The main ideas for this improvement depends on the accuracy of edge information provided. This condition helps the optimizer tools make a decision during cost computation. The accurate additional information avoids the estimation tool for predicting the same solution frequently. As a result, this edge enhancement transforms the IBP technique more efficient in predicting and light computational.

Gratefully, the proposed technique capable to boost up the Gradient Descent performance better than previous. In additional this technique can speed up the cost computational until $70 \%$ faster from the conventional Gradient Descent method. This situation proved that the edge enhancement technique assists to optimize the estimation process during seeking the final HR image. The accurate estimation function depends on the precision of edge detector provides the high localization and high rate edge detection. This accuracy edge detection helps the estimator to predict further step to getting close the optimal result. Table 2 shows the number of Iterations 
Table 2. Number of Iterations

\begin{tabular}{llllll}
\hline & \multicolumn{5}{c}{ The IBP technique with } \\
& IBP & Patel & Cheref & CANNY & SISEF \\
\hline LENA & 89 & 46 & 45 & 72 & 30 \\
CAMERAMAN & 82 & 46 & 44 & 67 & 27 \\
MANDRIL & 117 & 65 & 58 & 99 & 35 \\
PEPPER & 116 & 62 & 57 & 96 & 31 \\
CLOWN & 114 & 63 & 57 & 94 & 39 \\
JETPLANE & 92 & 47 & 48 & 76 & 35 \\
BRAIN & 91 & 51 & 44 & 73 & 22 \\
SHIP & 78 & 41 & 41 & 63 & 25 \\
USAF & 44 & 27 & 30 & 37 & 25 \\
BRIDGE & 98 & 56 & 50 & 81 & 34 \\
\hline
\end{tabular}

Another key point for speed up the estimation depends on the accurate rate of step size for the gradient. In reality, the gradient value detected varies large intensity amount, thus the estimation never be converging after receiving the large amount of data. This situation can be handled by sets the suitable rate for step size. The selection of step size is important to ensure estimator receives an optimum information with less computational cost. In this experiment all technique examines provided same optimum step size expect the technique proposed by Patel. Thus, this estimation efficiency totally depends on the performance of edge detector performance. The result in Table 4.4 shown, the Sharp ISEF edge detector is the best regulator during performing the estimation process since this technique provides lesser iteration compare the other regulator technique. Additionally, the proposed technique and the Patel technique use same edge detector. However, the suitable step size rate gives an advantage of proposed techniques to become light cost complexity up to $40 \%$ faster.

\section{CONCLUSION}

This study proposed a precise edge enhancement regulator to improve the IBP reconstruction technique problem by using the Sharp ISEF regularization. Normally, the IBP reconstruction technique has suffered from produced the ringing artefacts on HR estimation and it also required a large number of iterations to accomplish the estimation. Moreover, this technique leak of capability to preserve the lost high frequency information due to blurry effect. However, the Sharp ISEF has a solution to cure the IBP reconstruction problem with providing precise edge enhancement with light cost computation. The Sharp ISEF method has highly precision localization and less error rate detection. Thus these advantages help the estimator to compute the correction process more accurate and faster.

\section{ACKNOWLEDGEMENTS}

The author would like to acknowledge the support from the Fundamental Research Grant Scheme (FRGS) under a grant number of FRGS/1/2017/ICT05/UNIMAP-/02/2 from the Ministry of Higher Education Malaysia. The author would like to express gratitude to the Ministry of Higher Education Malaysia and University Malaysia Perlis for facilities provided in this work.

\section{REFERENCES}

[1] M.-C. Chiang and T. E. Boult, "Efficient image warping and super-resolution," Applications of Computer Vision, 1996. WACV '96., Proceedings 3rd IEEE Workshop on. pp. 56-61, 1996.

[2] B. Cohen and I. Dinstein, "Polyphase back-projection filtering for image resolution enhancement," IEE Proc. Vision, Image, Signal Process., vol. 147, no. 4, p. 318, 2000.

[3] Hyunwoo Kim, Jeong-Hun Jang, and Ki-Sang Hong, "Edge-enhancing super-resolution using anisotropic diffusion," in Proceedings 2001 International Conference on Image Processing (Cat. No.01CH37205), 2001, vol. 2, pp. 130-133.

[4] Z. Yan, Y. Lu, and H. Yan, "Reducing The Spiral CT Slice Thickness Using Super Resolution," 2010 IEEE International Conference on Image Processing. pp. 593-596, 2010.

[5] F. Qin, "An improved super resolution reconstruction method based on initial value estimation," 2010 3rd Int. Congr. Image Signal Process., pp. 826-829, 2010.

[6] X. Liang and Z. Gan, "Improved Non-local Iterative Back-Projection Method for Image Super-Resolution," 2011 Sixth Int. Conf. Image Graph., pp. 176-181, 2011.

[7] T. Bengtsson, I. Y. H. Gu, M. Viberg, and K. Lindström, "Regularized optimization for joint super-resolution and high dynamic range image reconstruction in a perceptually uniform domain," 2012 IEEE International Conference on Acoustics, Speech and Signal Processing (ICASSP). pp. 1097-1100, 2012.

[8] M. N. Bareja, “An Effective Iterative Back Projection based Single Image Super Resolution Approach,” 2012.

Edge enhancement of IBP reconstruction by using sharp infinite symmetrical exponential... (A.R.A Nazren) 
[9] R. Nayak, S. Monalisa, and D. Patra, "Spatial super resolution based image reconstruction using HIBP," in 2013 Annual IEEE India Conference (INDICON), 2013, pp. 1-6.

[10] P. Rasti, H. Demirel, and G. Anbarjafari, "Image resolution enhancement by using interpolation followed by iterative back projection," 2013 21st Signal Processing and Communications Applications Conference (SIU). pp. 1-4, 2013.

[11] Y. Cheref and D. Yousfi, "Image reconstruction with better edge enhancement using super resolution algorithm," in 2014 International Conference on Multimedia Computing and Systems (ICMCS), 2014, pp. 283-288.

[12] J. Mei, H. Gao, B. Maiseli, and O. Elisha, "Edge preservation image enlargement and enhancement method based on the adaptive Perona-Malik non-linear diffusion model," IET Image Process., vol. 8, no. 12, pp. 753-760, 2014.

[13] X. Yang, Y. Zhang, D. Zhou, and R. Yang, “An improved iterative back projection algorithm based on ringing artifacts suppression," Neurocomputing, vol. 162, pp. 171-179, 2015.

[14] M. Nawaz, R. Xie, L. Zhang, M. Asfandyar, and M. Hussain, "Image super resolution by sparse linear regression and iterative back projection," 2016 IEEE International Symposium on Broadband Multimedia Systems and Broadcasting (BMSB). pp. 1-6, 2016.

[15] A. R. A. Nazren, S. N. Yaakob, R. Ngadiran, M. B. Hisham, and N. M. Wafi, "Improving iterative back projection super resolution model via anisotropic diffusion edge enhancement," in 2016 International Conference on Robotics, Automation and Sciences (ICORAS), 2016, pp. 1-4.

[16] R. R. Makwana and N. D. Mehta, Emerging Trends in Electrical, Electronic and Communications Engineering, vol. 416, no. 1. Cham: Springer International Publishing, 2017.

[17] R. Nayak and D. Patra, "An edge preserving IBP based super resolution image reconstruction using P-spline and MuCSO-QPSO algorithm,” Microsyst. Technol., vol. 23, no. 3, pp. 553-569, Mar. 2017.

[18] M. Irani, M. Irani, S. Peleg, and S. Peleg, "Motion Analysis for Image Enhancement:Resolution, Occlusion, and Transparency," J. Vis. Commun. Image Represent., vol. 4, pp. 324-335, 1993.

[19] S. Rathore and Y. Harshalatha, "Iterative Back-Projection Algorithm Based Signal Processing Approach To Enhance An Image Resolution," Int. J. Eng. Res. Appl., vol. 2, no. 6, pp. 1449-1454, 2012.

[20] S. Castan, J. Zhao, and J. Shen, "New edge detection methods based on exponential filter," in [1990] Proceedings. 10th International Conference on Pattern Recognition, 1990, vol. i, no. X, pp. 709-711.

[21] P. S. A. Patel Shreyas A., "Novel Iterative Back Projection Approach," IOSR J. Comput. Eng., vol. 11, no. 1, pp. 65-69, 2013

\section{BIOGRAPHIES OF AUTHORS}
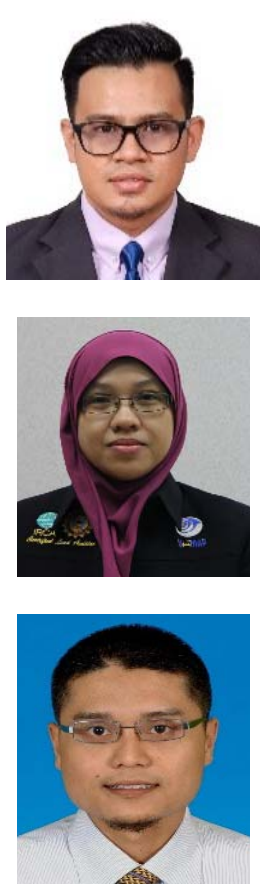

First Author received the B. Engineering (Hons) in Computer Engineering from University Malaysia Perlis (UniMAP), Malaysia and Ph.D. degree in the Computer Engineering from University Malaysia Perlis, Malaysia.

Second Author received the B. Engineering (Hons) in Communication and Computer Engineering from University Kebangsaan Malaysia (UKM), Malaysia. and the M.S. degree in Computer from University Kebangsaan Malaysia (UKM), in 2006, and Ph.D. degree in Communications, Sensors and Signal Processing from Newcastle University, UK.

Third Author received the B.Eng. in Electrical-Electronics (Hons) from University Teknologi Malaysia (UTM) and M.S. degrees by research in Computer Engineering from University Malaysia Perlis (UniMAP) the Ph.D. degree in the Computer System Engineering from University South Australia, Australia. 